\title{
On the role of tyrosine phosphatases as negative regulators of STAT signaling in breast cancers: new findings and future perspectives
}

\author{
Michel L Tremblay*
}

\begin{abstract}
The increasing importance of signal transducer and activator of transcription 3 (STAT3) expression in human cancers has led several laboratories to examine in detail the expression of one of its major negative regulators in oncogenesis - the T-cell protein tyrosine phosphatase, nonreceptor type 2 (PTPN2). A recent paper by Shields and colleagues points to the associate depletion of PTPN2 in STAT3-positive breast cancers. We examine these findings and reflect on the mechanism of action of PTPN2 and the consequences of its modulation for STAT3 downstream signaling.
\end{abstract}

Research on breast cancer and associated therapies have been at the forefront of personalized medicine, particularly with the help of high-throughput genomic technologies and sophisticated computational analysis. The most obvious outcome is the discrimination between distinct cancer subtypes and the increasing number of specific markers that reflect the heterogeneity of breast cancers [1].

An important predictor of negative prognosis is signal transducer and activator of transcription 3 (STAT3) [2], a pleiotropic transcription factor phosphorylated by several tyrosine kinases that are specifically activated in oncogenesis [3]. The negative regulation of Janus kinase (JAK)-STAT signaling is often overlooked despite being essential for balancing target gene expression output in response to external cues. Together with the well-known suppressors of cytokine signaling and protein inhibitors of activated STATs, protein tyrosine phosphatases (PTPs) are also key negative modulators of JAK-STAT signaling as recently reviewed, for instance, in the downstream

*Correspondence: michel.tremblay@mcgill.ca

Room 613, Cancer Building, McGill University, 1160 Pine Avenue, Montreal, Quebec, Canada H3A 1A3 modulation of leptin signaling [4]. Several cancers present high levels of phospho-STAT3, and the dysregulation of the negative regulators of JAK-STAT signaling have been found to be the underlying cause in a number of cases. T-cell protein tyrosine phosphatase, nonreceptor type 2 (PTPN2) was identified as a negative modulator of STAT3 in 2002 by Shuai's laboratory [5] and its homozygous deletion was later found to be responsible for a subtype of T-cell acute lymphoblastic leukemia [6]. Indeed, every case of cancer recurrence in this subtype of T-cell acute lymphoblastic leukemia was consistently associated with the homozygotic loss of PTPN2 gene.

Building on these important findings, the recent paper by Shields and colleagues examines the role of T-cell protein tyrosine phosphatase (TC-PTP) in breast cancer [7]. Using immunocytochemistry the authors found that $\sim 20 \%$ of all breast tumors present little to no TC-PTP, and that the lack of TC-PTP characterizes over twothirds of human breast primary tumor cells when triplenegative tumors are interrogated. Although the authors do not investigate the cause of the TC-PTP depletion, this finding may provide a useful, new molecular prognostic marker for the most severe type of triple-negative breast tumors.

Notably, Shields and colleagues also examined the mechanism of action of TC-PTP in modulating STAT3. First they showed that the (mainly nuclear) TC-45 isoform of TC-PTP is the principal negative regulator of STAT3 downstream of the epidermal growth factor receptor, and that the knockdown of TC-PTP does not lead to increased epidermal growth factor receptor tyrosine phosphorylation nor phosphatidylinositol 3-kinase or mitogen-activated protein kinase signaling. However, higher phosphorylation levels of the Src kinase (Y418) and STAT3 (Y705) were detected, thus supporting their identities as TC-PTP substrates. Interestingly, the authors also reported that IL-6-stimulated JAK1 is hyperphosphorylated in HeLa cells in TC-PTP knockdowns. In contrast, this is not the case in other breast cancer cell lines interrogated (T-47D, SK-BR-3 and HCC-1954) where, although STAT3 becomes hyperphosphorylated 
in the absence of TC-PTP, JAK1 and epidermal growth factor receptor seemingly are not. Moreover, this regulation of STAT3 is nicely shown by the Tiganis group to be cell and signaling context dependent, since the increased phosphorylation of STAT3 and Src family protein tyrosine kinase following plating on collagen is not significantly affected by the overexpression of TC-PTP [7]. An important conclusion to these intriguing results is that the targeting of these substrates by TC-PTP is not a random housekeeping event, but one that is selectively controlled, even in experiments where TC-PTP is overexpressed.

Overall, Shields and colleagues bring important points on the contribution of TC-PTP in the negative regulation of STAT3 in solid tumors. An unbiased search in Drosophila has previously shown that the most important negative regulator of JAK-STAT is DPTP61F, the fly ortholog of human TC-PTP (PTPN2) and PTP1B (PTP nonreceptor type 1) [8]. Moreover, a recent study by the Miranda-Saavedra group where multiple STAT3 ChIP-seq libraries prepared from distinct cell types were compared showed that the genome-wide binding patterns of STAT3 are largely cell type specific [9]. However, a conserved core of STAT3-dependent target genes transcription $(n=35)$ is shared in all cell types investigated. This universal and cell type-independent regulatory network includes STAT3's self-regulation, as well as STAT3mediated transcription of negative regulators such as PTP1B and suppressor of cytokine signaling 3 . If these findings can be extrapolated to breast cancer, this will mean that STAT3 activation in breast cancer may cause a diversity of transcriptional responses depending on the breast cancer subtype, and that cancer cells expressing hyperphosphorylated STAT3 may also need to silence some or all STAT3-regulated negative feedback loops for tumor cells to remain oncogenic.

An additional and critical question here is the ultimate consequences of PTPs in the negative regulation of phospho-STAT3, both in normal epithelial cells and in breast cancer cells: is TC-PTP as well as other PTPs simply acting as a rheostat - that is, a quantitative regulator of JAK-STAT signaling? Or do different levels of PTPs lead to quantitative as well as qualitative changes in the downstream STAT3 target genes? PTPs are often seen as off-switches in protein phosphorylation, yet they modulate both positively and negatively a large number of pathways. The findings reported by Shields and colleagues, and those of Hutchins and colleagues using a system immunology approach, are slowly unveiling new and important regulatory mechanisms controlled by PTPs. These mechanisms likely have a significant role in STAT3-promoted breast cancers. It will be exciting to see a better understanding of these mechanisms in normal and cancer cells with the rapid integration of biochemical and cellular biology with high-throughput genomics and bioinformatics methodology.

\section{Abbreviations}

IL, interleukin; JAK, Janus kinase; PTP, protein tyrosine phosphatase; PTPN2, protein tyrosine phosphatase, nonreceptor type 2; STAT3, signal transducer and activator of transcription 3; TC-PTP, T-cell protein tyrosine phosphatase.

\section{Competing interests}

The author declares that he has no competing interests.

\section{Acknowledgements}

The author is grateful to collaborator Diego Miranda-Saavedra (IFReC) and the members of the Tremblay Laboratory, Kelly Pike and Stephanie MarmenBussieres for their comments on this manuscript. MLT is a holder of the Jeanne and Jean-Louis Lévesque Chair in Cancer Research.

Published: 31 July 2013

\section{References}

1. Landis MD, Lehmann BD, Pietenpol JA, Chang JC: Patient-derived breast tumor xenografts facilitating personalized cancer therapy. Breast Cancer Res 2013, 15:201.

2. Sonnenblick A, Uziely B, Nechushtan H, Kadouri L, Galun E, Axelrod JH, Katz D, Daum H, Hamburger T, Maly B, Allweis TM, Peretz T: Tumor STAT3 tyrosine phosphorylation status, as a predictor of benefit from adjuvant chemotherapy for breast cancer. Breast Cancer Res Treat 2013, 138:407-413.

3. Yu H, Pardoll D, Jove R: STATs in cancer inflammation and immunity: a leading role for STAT3. Nat Rev Cancer 2009, 9:798-809.

4. St-Pierre J, Tremblay ML: Modulation of leptin resistance by protein tyrosine phosphatases. Cell Metab 2012, 15:292-297.

5. ten Hoeve J, de Jesus Ibarra-Sanchez M, Fu Y, Zhu W, Tremblay M, David M, Shuai K: Identification of a nuclear Stat1 protein tyrosine phosphatase. Mol Cell Biol 2002, 22:5662-5668.

6. Kleppe M, Lahortiga I, El Chaar T, De Keersmaecker K, Mentens N, Graux C, Van Roosbroeck K, Ferrando AA, Langerak AW, Meijerink JP, Sigaux F, Haferlach T, Wlodarska I, Vandenberghe P, Soulier J, Cools J: Deletion of the protein tyrosine phosphatase gene PTPN2 in T-cell acute lymphoblastic leukemia. Nat Genet 2010, 42:530-535.

7. Shields BJ, Wiede F, Gurzov EN, Wee K, Hauser C, Zhu HJ, Molloy TJ, O'Toole SA, Daly RJ, Sutherland RL, Mitchell CA, McLean CA, Tiganis T: TCPTP regulates SFK and STAT3 signaling and is lost in triple-negative breast cancers. Mol Cell Biol 2013, 33:557-570.

8. Baeg GH, Zhou R, Perrimon N: Genome-wide RNAi analysis of JAK/STAT signaling components in Drosophila. Genes Dev 2005, 19:1861-1870.

9. Hutchins AP, Diez D, Takahashi Y, Ahmad S, Jauch R, Tremblay ML, Miranda-Saavedra D: Distinct transcriptional regulatory modules underlie STAT3's cell type-independent and cell type-specific functions. Nucleic Acids Res 2013, 41:2155-2170.

doi:10.1186/bcr3437

Cite this article as: Tremblay ML: On the role of tyrosine phosphatases as negative regulators of STAT signaling in breast cancers: new findings and future perspectives. Breast Cancer Research 2013, 15:312. 